\title{
A HEVC Steganalysis Algorithm Based on Relationship of Adjacent Intra Prediction Modes
}

\author{
Henan Shi \\ Shanghai Jiao Tong University \\ Tanfeng Sun ( $\nabla$ tfsun@sjtu.edu.cn ) \\ Shanghai Jiao Tong University \\ Zhaohong Li \\ Beijing Jiaotong University
}

\section{Research}

Keywords: Video steganalysis, HEVC, Intra Prediction Mode (IPM), Most Probable Mode (MPM), GrayLevel Co-occurrence Matrix (GLCM).

Posted Date: September 4th, 2020

DOl: https://doi.org/10.21203/rs.3.rs-67799/v1

License: (c) (1) This work is licensed under a Creative Commons Attribution 4.0 International License. Read Full License 


\section{Intra Prediction Modes}

${ }^{1}$ School of Electronic Information and Electrical Engineering, Shanghai Jiao Tong University, 800,

Dongchuan Road, Minhang District, Shanghai, China

${ }^{2}$ School of Electronic and Information Engineering, Beijing Jiaotong University, 3, Shangyuancun,

Haidian District, Beijing, China

8 Correspondence should be addressed to Tanfeng Sun, tfsun@sjtu.edu.cn

\section{Abstract}

10 Currently, many High Efficiency Video Coding (HEVC) video steganography algorithms based on Intra

11 Prediction Mode (IPM) have been proposed. However, the existing IPM-based video steganalysis

12 algorithms are almost designed for H.264/AVC videos, without considering the unique coding techniques

13 in HEVC, which is the latest video codec standard. Thus, it is of significant value to study IPM-based

14 steganalysis for HEVC videos. In this paper, the general process of IPM-based HEVC steganography is

15 modelled for the first time, and we find that the basic distortion existing in the change of the relationships

16 between each embedded IPM and the adjacent IPMs. By exploiting these weaknesses, we propose a

17 novel IPM steganalysis algorithm based on the Relationship of Adjacent IPMs (RoAIPM) feature. In

18 detail, the RoAIPM is extracted by generating different directional Gray-Level Co-occurrence Matrixes

19 (GLCMs) and texture characteristics of three refilled matrixes: MPM-IPM matrix, Left-IPM matrix and

20 Up-IPM matrix. Experimental results show that, the proposed RoAIPM feature is very sensitive to the

21 little change introduced by IPM-based steganography. Regardless of whether the feature is after 
1 dimension reduction or not, in various coding conditions, the proposed steganalysis can both present a

2 well higher detection accuracy against the latest IPM-based HEVC steganography methods and achieve

3 the lowest computational complexity compared with the state-of-the-art works.

4 Keywords: Video steganalysis, HEVC, Intra Prediction Mode (IPM), Most Probable Mode (MPM),

5 Gray-Level Co-occurrence Matrix (GLCM).

\section{1. Introduction}

7 Steganography is an art and science of covert communication by hiding messages into universal digital

8 media without raising suspicion. In modern steganography, while stego-objects are modified in some

9 parameter characteristics, the human eye cannot distinguish stego-objects from cover-objects. As a

10 counter-technique to steganography, steganalysis aims to detect the presence of hidden data in digital

11 media. The development of steganalysis takes an immeasurable effect to cope with the abuse of

12 steganography and strengthen national security. Thus, steganalysis has also been widely studied.

13 In modern steganography, digital media carriers can be images, videos and documents etc. Digital

14 video, as an emerging mainstream media exploding on the network, has the characteristics of high

15 capacity and insensitivity to distortion. Obviously, using videos as carriers of secret messages has more

16 advantages than other objects. Besides, High Efficiency Video Coding (HEVC) is the newest video

17 coding standard, which significantly improves compression performance and will be more widely

18 adopted relative to H.264/AVC [1]. Consequently, the research on steganalysis for HEVC videos is of

19 important academic significance and high applied value.

20 According to the embedding domain, video steganography can be classified into spatial domain

21 steganography and compressed domain steganography. The first type embeds messages in pixels. The 
second type embeds data by modifying compressed domain parameters, such as Motion Vectors (MVs) prediction modes [14-17]. Since videos are transmitted mostly in compressed format, the second type is more practical. In the intra coding process of HEVC, some video steganography schemes based on Intra Prediction Modes (IPMs) have appeared. As a result of information embedding, modified IPMs are no longer optimal. These steganography methods generally have considerable capacity of secret information and low computational complexity. According to the distortion introduced by modifying IPMs in intra coding process of HEVC, Dong et al. [14] presented a HEVC Steganographic Channel Model (SCM), and proposed a novel coding efficiency preserving steganography algorithm based on Prediction Units

10 (PUs), which took the IPMs of candidate PUs as cover. Later, Dong et al. [15] optimized the previous

11 algorithm, instead of using single-sized blocks, such as PUs with size of $4 \times 4$, to embed secret data, it 12 exploited IPMs in multi-sized Prediction Blocks (PBs) in each Coding Tree Unit (CTU), achieving the 13 improvement in capacity without introducing significant degradation in visual quality while preserving 14 coding efficiency as well. Wang et al. [16] gave an effective IPM-based video steganography for HEVC 15 to maintain rate-distortion optimization and increase empirical security. This algorithm captured the embedding impacts on both neighboring and current PUs by designing a special distortion function, and

17 three-layered isolated channels were established according to the property of IPM coding. Besides, Wang et al. [17] also designed a novel video steganography based on IPM for HEVC, which maintained video

19 quality and improved security performance. It presented the probability distribution of $4 \times 4$ IPMs, and proposed a cover selection rule combined with Coding Units (CUs) and PUs coding information. These 
steganography methods can maintain good visual quality, which makes it hard to detect the stego-videos by previous steganalysis algorithms.

However, to the best knowledge of the authors, there are few video steganalysis algorithms that can effectively detect IPM-based HEVC steganography, and thus IPM-based steganography algorithms in HEVC lack targeted security detection. Currently, the widely used IPM-based video steganalysis algorithm with a better performance was proposed by Zhao et al. [18]. It proposed a calibration-based video steganalysis, and extracted IPM Calibration (IPMC) features to detect the steganography. Although the features were of a low dimension and sensitive to the changes of IPMs, this algorithm was specially designed for H.264/AVC videos, and it did not consider the uniqueness and innovation of HEVC video coding. Therefore, when detecting IPM-based HEVC steganography, the effect will be greatly reduced. By analyzing modified IPMs are no longer optimal in the existing IPM-based HEVC steganography,

12 we propose a novel IPM steganalysis algorithm based on the Relationship of Adjacent IPMs (RoAIPM)

13 features for HEVC videos. During the process of intra-coding, in order to ensure the quality of 14 compressed videos and minimize the size of bit stream, the IPMs in original videos are usually the 15 optimal IPMs in accordance with the intra-modes coding which defines three Most Probable Modes 16 (MPMs) for current luma PU. However, the IPMs of the modified blocks in such stego-videos are 17 generally not the optimal. In fact, the contents of neighboring image blocks are similar, and thus their 18 optimal IPMs are close. But the characteristic that the IPMs of neighboring blocks are close is destroyed 19 by the embedding of secret information. On the other hand, in HEVC intra prediction process, the MPMs 20 of current luma PU are decided by the IPMs of neighboring encoded luma PBs. Thus, in the stego-videos, 21 the probability that the IPM of current block belongs to the three MPMs is greatly reduced. If we present 
1 the IPMs distribution of neighboring blocks and the MPMs of every current block in the form of matrix,

2 and then capture the relationships between the IPM of each embedded block and the adjacent IPMs as

3 well as its MPMs, the steganalysis algorithm will obtain fundamental feature changes of stego-videos.

4 According to this principle, the algorithm captures the RoAIPM features by adopting Gray-Level Co-

5 occurrence Matrix (GLCM), which can reflect the change of the relationship between adjacent IPMs in

6 different directions, so as to achieve the goal of detecting the stego-videos. Although the universality of

7 features from all blocks of different sizes is better, they are less targeted and less sensitive to such

8 steganography, together with the fact that $4 \times 4$ PUs are main covers in all IPM-based steganography, so

9 the RoAIPM features are extracted only from $4 \times 4$ PUs. Finally, the features will be sent into Support

10 Vector Machine (SVM) to classify stego-videos and cover-videos. Experimental results demonstrate that

11 the RoAIPM is sensitive to the modification of IPMs, and the accuracy rates are higher than existing

12 other algorithms. Besides, the proposed algorithm has the lowest computational complexity compared

13 with other works, and thus it is easier to implement.

14 The rest of this paper is organized as follows: In Section 2, the HEVC intra prediction process is

15 introduced, and the general model of IPM-based HEVC steganography is analyzed. In Section 3, the

16 producing principle of the RoAIPM features is explained. The proposed steganalysis algorithm is

17 described in detail in Section 4. Next, Section 5 shows the experimental results and analysis. Finally, the

18 conclusions and future work are given in Section 6.

\section{2. Modeling of IPM-Based HEVC Steganography}


1 In order to keep this paper more self-contained, the basics of intra prediction in HEVC are introduced

2 in Section 2.1. Then, the specific process of IPM-based HEVC steganography is analyzed and modeled

3 for the first time in Section 2.2.

\subsection{Basics of Intra Prediction in HEVC}

HEVC adopts the hybrid coding framework used in H.264/AVC. There are many innovative technologies and concepts introduced. For instance, instead of the concept of the fixed size macroblock in H.264/AVC, the size of blocks in HEVC can be changed self-adaptively. HEVC defines a set of brand new concepts

8 for block coding, including Coding Tree Unit (CTU), Coding Unit (CU), Prediction Unit (PU) and

9 Transform Unit (TU). The flexibility of HEVC coding, such as transform and prediction, depends much on these elements. A frame in HEVC is first spilt into several $64 \times 64$ nonoverlapping CTUs. Then every

11 CTU can either be directly used as CU or be further partitioned into multiple CUs with sizes of $32 \times 32$,

$1216 \times 16$ or $8 \times 8$. In the smooth region of a frame, the sizes of CUs are larger, and the smaller CUs are

13 selected in the edge or the area with complex texture, which is beneficial to improve coding efficiency.

14 CUs can be further split into PUs and TUs. An intra predicted CU may have one of the two types

15 of PU partitions modes: $2 N \times 2 N$ and $N \times N$. The first type means that the $\mathrm{CU}$ is not split, and the second

16 type indicates that the $\mathrm{CU}$ is split into four equal-sized PUs. PUs are the blocks where Intra Prediction

17 Modes (IPMs) are established, and each PU has its own IPM. Intra-prediction aims to remove spatial

18 redundancies between the current block and its neighbors. Compared with H.264/AVC, the number of

19 IPMs increases from 9 to 35 in HEVC, including planar mode numbered 0, DC mode numbered 1 and

2033 angular modes defined for luma information, as shown in Figure 1 [19]. 


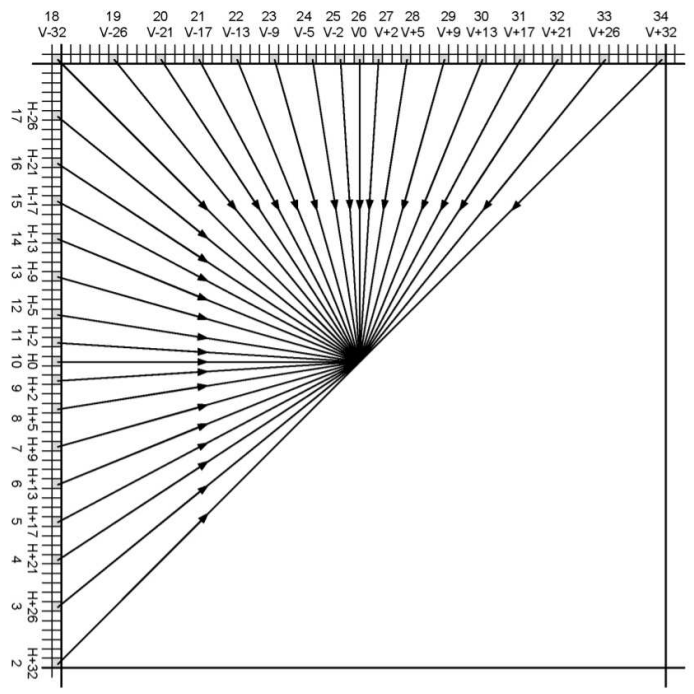

Figure 1: Angular intra-prediction modes numbered from 2 to 34 in HEVC. choose several candidate modes from the 35 modes. The number of candidate modes is determined by the size of the PU. RMD will refer to the Sum of Absolute Transformed Difference (SATD) and the length of bits used for expressing the coding information of each mode. Secondly, three Most Probable Modes (MPMs) will be added into above candidate set. If the current IPM is one of the three MPMs, only its index in the MPMs needs to be encoded. Otherwise, its index in remaining 32 modes needs to be encoded by using a code of 5-bit fixed length. The MPMs are decided by the IPMs of the upper and left encoded luma PUs, and if the upper and left PUs are unavailable, they will be set DC mode for

11 calculating MPMs. As depicted in Figure 2, $m_{\text {left }}$ is the coding mode number of the left (A) encoded PB and $m_{\text {upper }}$ is the coding mode number of the upper (B) encoded PB. Then three MPMs of $m_{\text {current }}$

13 are set according to $m_{\text {left }}$ and $m_{\text {upper }}$. The concrete calculation rule can be learned from Sullivan et 14 al. [1]. Finally, Rate Distortion Optimization (RDO) technique is adopted to calculate the RD cost of candidate modes. The mode with the minimum RD cost is selected as the optimal mode of the PU. 

covers, and the following IPMs specially refer to $4 \times 4$ IPMs. Figure 3 describes a general embedding 11 process of IPM-based HEVC steganography.

\subsection{Modeling of IPM-Based HEVC Steganography}

In this section, the specific process of IPM-based HEVC steganography is analyzed and modeled for the first time. As already mentioned, though there is the algorithm using IPMs in multi-sized PBs for information embedding to improve the capacity, such as Dong et al. [15], texture rich regions are more suitable for steganography and most of existing IPM-based steganography algorithms only choose the PBs with size of $4 \times 4$ as embedding covers. Therefore, the proposed steganalysis only extracts the features based on $4 \times 4 \mathrm{PBs}$, which is also effective against the steganography using multi-sized PBs as

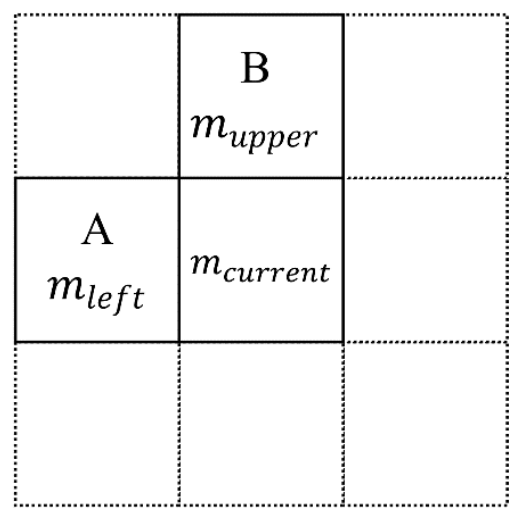

Figure 2: Reference blocks of MPMs. 


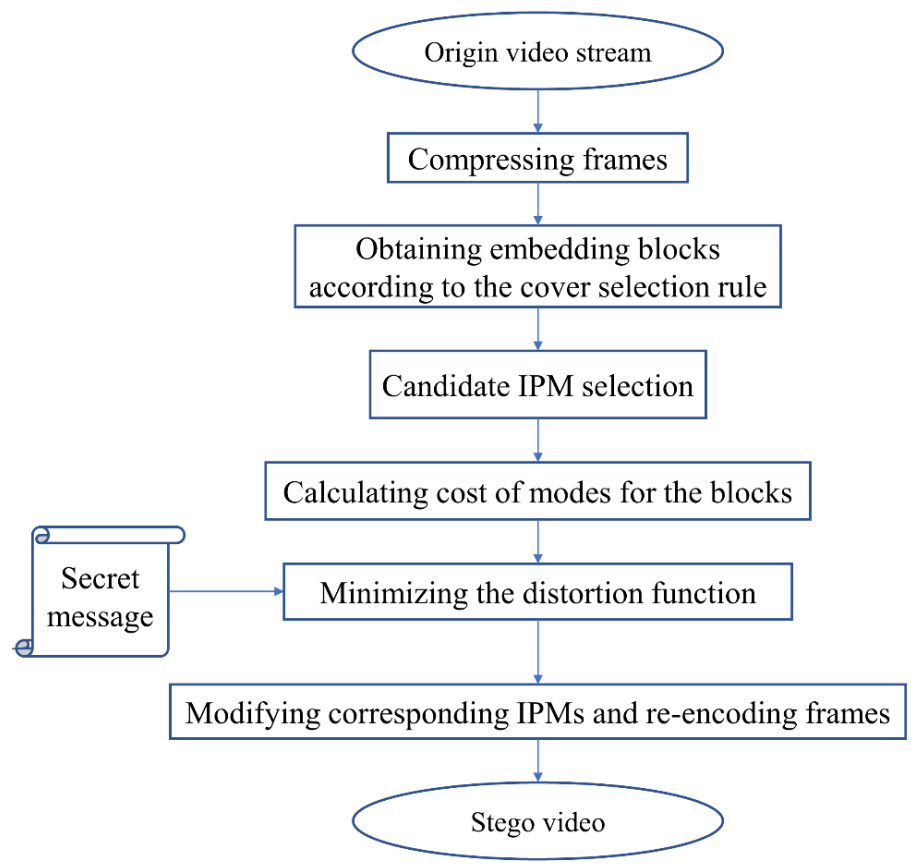

Figure 3: General embedding process of IPM-based HEVC steganography.

Firstly, compress the frames read from original video stream using HEVC encoder. Then, obtain

4 embedding blocks as well as theirs IPMs according to the cover selection rule. In HEVC, two number-

5 adjacent blocks have similar prediction directions, and the principle of modifying IPMs is replacing them

6 with the modes that are similar in prediction direction. In different algorithms, one prediction mode has

7 one or several candidate IPMs that can replace it. In current IPM-based steganography, in order to reduce

8 the influence on coding efficiency and enhance security, concrete coding methods of secret message are

9 mainly based on the Syndrome-Trellis Code (STC). Detailed illustration and implement of STC can be

10 found in Filler et al. [20]. By calculating Rate Distortion (RD) cost of the candidate and original modes

11 for each embedding block, the cost of IPM changing can be obtained, and the sum of total cost is the

12 final distortion function. In addition, in original videos, the IPM that yields the minimum RD cost will

13 be chosen as the optimal one to predict and encode current PU. Finally, use the modified IPMs that

14 minimize the final distortion function to re-encode the current frame. 
2 the modes that yield the minimum cost for IPMs changing. IPM-based steganography may divide 35

3 modified IPMs into two groups. One represents secret data 0 , and the other represents secret data 1 .

4 Steganography operation can be generally expressed as follows:

$$
\widetilde{m}_{k}=m_{k}+\beta_{k} \cdot \omega_{k}
$$
denotes the original IPM of block $k . \omega_{k} \in[-34,34]$ indicates the change introduced by steganography. Above parameters are all integers. Besides, $\beta_{k} \in\{0,1\}$ determines whether block $k$ is used to embed information.

The main distortion caused by this steganography is the reduction in coding efficiency. The

11 distortion on visual quality is very weak when the video bit rate is sufficiently high, and thus it is not

12 feasible to detect the visual quality distortion caused by IPM-based steganography. Through analysis, we

13 can know that the steganography usually modifies IPMs with non-optimal selection rules. But even if

14 the modified IPM of each embedded block is very close to the original one, in consideration of the

15 similarity of adjacent IPMs in cover-videos, this type of embedding method will also break the IPM-

16 similarity between adjacent blocks. Therefore, we focus more on the change of relationships between

17 adjacent blocks to detect steganography. adjacent upper block is expressed as Equation (2),

$$
\varphi_{k-\text { upper }}=\left|\operatorname{dist}\left(\widetilde{m}_{k}, m_{\text {upper }}\right)-\operatorname{dist}\left(m_{k}, m_{\text {upper }}\right)\right|,
$$


1 where $m_{\text {upper }} \in[0,34]$ denotes the IPM of the upper block. Function $\operatorname{dist}(x, y)$ represents the

2 absolute value of the difference between number $x$ and $y$. In original videos, the contents of neighboring

3 coding blocks are similar, and thus the value of $\operatorname{dist}\left(m_{k}, m_{\text {upper }}\right)$ may be smaller. Thus, $\varphi_{k-u p p e r}$

4 indicates the deviation degree of current IPM from the upper IPM. For the same reason, the distortion in

5 the distance between current IPM and the adjacent left IPM is defined as following formula:

$$
\varphi_{k-l e f t}=\left|\operatorname{dist}\left(\tilde{m}_{k}, m_{\text {left }}\right)-\operatorname{dist}\left(m_{k}, m_{\text {left }}\right)\right|
$$

Furthermore, for each I-picture, the total distortion $D_{\text {upper-left }}$ can be presented as Equation (4), where $n$ indicates the total number of all PUs that are embedded secret message in one I-picture.

$$
D_{\text {upper-left }}=D_{\text {upper }}+D_{\text {left }}=\sum_{k=1}^{n} \varphi_{k-\text { upper }}+\sum_{k=1}^{n} \varphi_{k-l e f t} .
$$

Based on the above analysis, we can conclude that: (1) IPM-based HEVC steganography destroys

11 the IPM-similarity of each embedded block and its adjacent blocks. (2) The probability that embedded

12 IPM is one of the MPMs is reduced. Taking advantage of these phenomenon, we establish the RoAIPM

13 features that can reflect the total distortion $D_{\text {upper-left }}$ and the violation of intra-modes coding in

14 accordance with three MPMs. It is worth mentioning that the distortion is weak and distributed, which

15 increases the difficulty of detection, and thus we adopt Gray-Level Co-occurrence Matrix (GLCM) as

16 well as its texture properties, which can reflect the relationship between adjacent IPMs in different

17 directions, so as to capture these distortions fully and improve the effect of detection against IPM-based

18 stego-videos.

\section{3. Proposed RoAIPM Features}

20 In this part, we introduce the producing method of the proposed Relationship of Adjacent IPMs (RoAIPM)

21 feature, which is a kind of fusion feature. Obviously, these features are tiny and decentralized. If we can 
1 present the IPMs distribution of adjacent blocks and the MPMs of every current block in the form of

2 matrix, and then indicate the relationships between the IPM of every embedded block and the IPMs of

3 its adjacent blocks as well as the MPMs, the RoAIPM is naturally fully obtained.

4 Firstly, the formation of texture is due to the repeated appearance of gray distribution in spatial

5 position, and GLCM is a common method to describe texture by studying the spatial correction of gray

6 level. Based on the analysis of IPM-based HEVC steganography, GLCM can count the number of all

7 possible combinations of two adjacent IPMs in different directions. Therefore, GLCM of adjacent IPMs

8 (GoAIPM) is adopted to constitute the RoAIPM features, so as to exactly capture the total distortion

$9 \quad D_{\text {upper-left }}$ and the relationships between each embedded IPM and its MPMs.

10 GoAIPM is defined as the probability that the value of new IPM is $I P M_{j}$ when leaving a fixed IPM

11 whose value is $I P M_{i}$ with distance $d$ and direction $\theta . \theta \in\left\{0^{\circ}, 45^{\circ}, 90^{\circ}, 135^{\circ}\right\}$. It can be formulated as:

$$
\operatorname{GoAIPM}\left(I P M_{i}, I P M_{j}\right)=\left\{\left|C_{1}, C_{2}\right| \mid C_{1}=\mathrm{C}\left(x_{1}, y_{1}\right)=I P M_{i}, C_{2}=\mathrm{C}\left(x_{2}, y_{2}\right)=I P M_{j}\right\}
$$

13 where $C$ is original IPM-matrix with grayscale $N_{g}$, and GoAIPM of size $N_{g} \times N_{g}$ represents the feature

14 matrix of $C .\left|C_{1}, C_{2}\right|$ is the number of ordinal combination $\left(I P M_{i}, I P M_{j}\right)$, which denotes the coordinate

15 of GoAIPM. The distance and direction between coordinate $\left(x_{1}, y_{1}\right)$ and $\left(x_{2}, y_{2}\right)$ is $d$ and $\theta$

16 respectively. That is, the number of all possible combinations of previous and after IPMs can be

17 expressed in the form of a matrix. In the actual producing process of the GoAIPM feature, the size of

18 matrix $C$ is decided by the video resolution, and the value of element IPM is range from 0 to 34 . Thus,

19 the value of $N_{g}$ is 35 , which is the number of IPMs in HEVC. For ease of understanding, take $d=1$ and

$20 \theta=0^{\circ}$ for example, as illustrated in Figure 4, the matrix $C$ with only IPMs ranging from 1 to 8 is chosen

21 as the original IPM-matrix for simplicity, and the generation process of the GoAIPM feature of matrix 
$1 C$ is depicted in red. Furthermore, the original IPM-matrix is modified in IPM-based stego-videos, and

2 according to Section 2.2, the main distortion is the change of the number of all possible combinations of

3 two adjacent IPMs. Therefore, the proposed GoAIPM feature can effectively capture distortion.

\begin{tabular}{r|r|r|r|r|r|r|r|r|r|r|r|r|r|r|}
$\mathbf{1}$ & \multicolumn{2}{c}{$\mathbf{2}$} & $\mathbf{3}$ & $\mathbf{4}$ & $\mathbf{5}$ & $\mathbf{6}$ & $\mathbf{7}$ & $\mathbf{8}$ \\
\hline $\mathbf{1}$ & 1 & 5 & 6 & 8 \\
\hline 2 & 3 & 5 & 7 & 1 \\
\hline 4 & 5 & 7 & 1 & 2 \\
\hline 8 & 5 & 1 & 2 & 5 \\
\hline
\end{tabular}

Figure 4: GoAIPM feature generation process of matrix C.

For the matrix with similar IPM values, the value on the diagonal of GoAIPM will be larger. Conversely, for the matrix with fast IPM change, the value deviating from the diagonal of GoAIPM will be larger. Generally, some scalars can be used to characterize the textures of GoAIPM. Here we will introduce three texture characteristics of GLCM: Angular Second Moment (ASM), correlation and homogeneity.

ASM is the summation of squared elements in the GLCM. In our application, ASM of Adjacent IPMs (ASMoAIPM) is formed as one kind of texture characteristics in Equation (6),

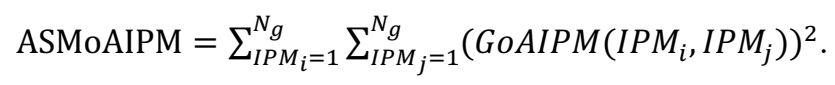

Correlation is the statistical measure of how correlated a parameter value is to its neighbor over the whole IPM-matrix, which reflects the consistency of texture, and Correlation of Adjacent IPMs (CORoAIPM) is used as another kind of texture characteristics to enrich the RoAIPM features. It is calculated as:

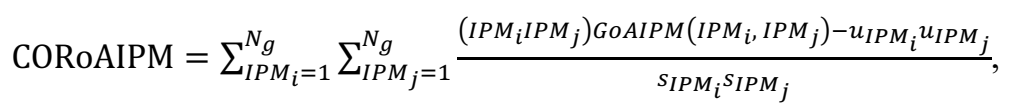

$$
u_{I P M_{i}}=\sum_{I P M_{i}=1}^{N_{g}} \sum_{I P M_{j}=1}^{N_{g}} I P M_{i} \cdot \operatorname{GoAIPM}\left(I P M_{i}, I P M_{j}\right),
$$




$$
\begin{gathered}
u_{I P M_{j}}=\sum_{I P M_{i}=1}^{N_{g}} \sum_{I P M_{j}=1}^{N_{g}} I P M_{j} \cdot \operatorname{GoAIPM}\left(I P M_{i}, I P M_{j}\right), \\
S_{I P M_{i}}{ }^{2}=\sum_{I P M_{i}=1}^{N_{g}} \sum_{I P M_{j}=1}^{N_{g}} \operatorname{GoAIPM}\left(I P M_{i}, I P M_{j}\right)\left(I P M_{i}-u_{I P M_{i}}\right)^{2}, \\
S_{I P M_{j}}{ }^{2}=\sum_{I P M_{i}=1}^{N_{g}} \sum_{I P M_{j}=1}^{N_{g}} \operatorname{GoAIPM}\left(I P M_{i}, I P M_{j}\right)\left(I P M_{j}-u_{I P M_{j}}\right)^{2} .
\end{gathered}
$$

Besides, homogeneity indicates the closeness of the distribution of elements in the GoAIPM to the

of Adjacent IPMs (HOMoAIPM) characteristic to present texture changes introduced by IPM-based steganography, which is formulated as Equation (12),

$$
\text { HOMoAIPM }=\sum_{I P M_{i}=1}^{N_{g}} \sum_{I P M_{j}=1}^{N_{g}} \frac{\operatorname{GoAIPM}\left(I P M_{i}, I P M_{j}\right)}{1+\left|I P M_{i}-I P M_{j}\right|} .
$$

Briefly, in view of the analysis that these texture characteristics can accurately capture the change of energy after steganography and the relationship between adjacent IPMs, in the proposed steganalysis

11 algorithm, three novel texture characteristics are also proposed to present the RoAIPM features. In conclusion, the proposed RoAIPM features include four parts: the GoAIPM feature, three texture characteristics of ASMoAIPM, CORoAIPM and HOMoAIPM, as following formula:

$$
\text { RoAIPM }=\{\text { GoAIPM, ASMoAIPM, CORoAIPM, HOMoAIPM }\}
$$

15 which ensure the sufficiency and effectiveness of the features reflecting the distortion on the relationships between each embedded IPM and the adjacent IPMs as well as its MPMs. Specific generation method of

17 the RoAIPM is presented in Section 4.

\section{4. Proposed Steganalysis Algorithm}

19 In this section, based on the above introduction of the RoAIPM features, we will illustrate the flow of the proposed IPM-based HEVC steganalysis algorithm concretely, and describe the specific generation 
2 Figure 5, and can be summarized in the following three steps:

11 videos by counting the number of all possible combinations of IPMs in different directions. However, the IPMs in a CU are probably same, which will weaken the expression of change of relationship between

13 adjacent IPMs, and the MPMs of each block need fill in adjacent positions, which is convenient for 14 GoAIPM to reflect the relationship of each IPM and its MPMs. Thus, three refilled matrixes are 
1 introduced to strengthen the statistics of embedding impact. Next, the three refilled matrixes will be

2 derived from the IPM matrix: MPM-IPM matrix, Left-IPM matrix and Up-IPM matrix according to

3 Equation (4). In these refilled matrixes, if the size of current PU is $4 \times 4$ and the four $4 \times 4$ PUs in one $8 \times 8$

4 CU have different IPMs, which can be classified as non-uniform $4 \times 4$ PUs, these four IPMs remain

5 unchanged, or else the IPM of current PU will be reset according to three different matrix filling rules.

6 As depicted in Figure 6, a small square represents a $4 \times 4$ block, and also provides a matrix element.

7 Taking uniform $4 \times 4$ PUs as example, which have the same IPM named $m_{\text {current }} . m_{\text {left }}$ is the IPM of

8 left adjacent block (A), and $m_{\text {upper }}$ is the IPM of upper adjacent block (B). MPM[0], MPM[1] and

9 MPM[2] are MPMs of $m_{\text {current }}$, which are set according to $m_{\text {left }}$ and $m_{\text {upper }}$. The reset IPMs in four

10 uniform $4 \times 4$ PUs in one $8 \times 8 \mathrm{CU}$ of each matrix are in blue. The IPMs of PUs of other bigger sizes are

11 refilled with the same rules. For the same reason, for the input video with resolution $1920 \times 1080$, the

12 sizes of MPM-IPM matrix, Left-IPM matrix and Up-IPM matrix are all $480 \times 270$.

13

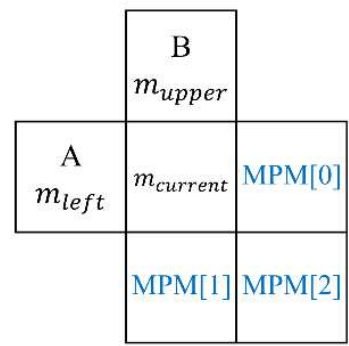

(a)

\begin{tabular}{|c|c|c|}
\hline $\begin{array}{c}\text { A } \\
m_{\text {left }}\end{array}$ & $m_{\text {current }}$ & $m_{\text {left }}$ \\
\hline & $m_{\text {current }}$ & $m_{\text {left }}$ \\
\cline { 2 - 3 } & &
\end{tabular}

(b)

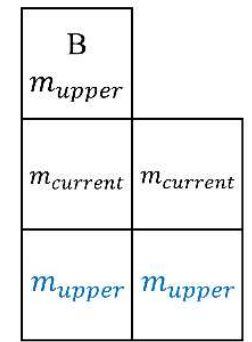

(c)

Figure 6: Filling rules of (a) MPM-IPM, (b) Left-IPM and (c) Up-IPM matrixes for uniform 4×4 PUs.

Secondly, due to the features from all blocks of different sizes are less targeted and sensitive to such steganography, as well as $4 \times 4$ PUs are main covers in all IPM-based steganography. Thus, the proposed RoAIPM features are extracted from $4 \times 4$ PUs by altering IPMs that are not in $4 \times 4$ PUs of the three matrixes to a non-existing value, such as 35 , and then deleting the row and column of this value in the 
1 GoAIPM. Specifically, according to different characteristics of the three matrixes, firstly generate the

2 GoAIPMs of MPM-IPM matrix with direction $\theta=0^{\circ}$ and $90^{\circ}$ respectively, and also obtain the three

3 texture characteristics of GoAIPM from MPM-IPM matrix: ASMoAIPM, CORoAIPM and HOMoAIPM,

4 with $\theta=0^{\circ}, 90^{\circ}$ and $135^{\circ}$. In the same way, generate the GoAIPMs of Left-IPM matrix and Up-IPM

5 matrix with $\theta=0^{\circ}$ and $90^{\circ}$ respectively, and also produce the three texture characteristics of GoAIPM

6 from Left-IPM matrix and Up-IPM matrix with $\theta=0^{\circ}$ and $90^{\circ}$ respectively. The parameter of

7 distance $d$ is always set as 1 . In this way, each GoAIPM has $35 \times 35$ features because of 35 IPMs in

8 HEVC, and thus the final RoAIPM features of 4915 dimensions are easy to establish.

9 In the process of generating the RoAIPM features, by using different parameters of direction in

10 GLCM to produce various GoAIPMs, we take full advantage of filling methods of three matrixes. IPMs

11 distribution of neighboring blocks and MPMs of every current block are more intuitively presented in

12 the three matrixes. Then, the changes of relationships between the IPM of every embedded block and the

13 IPMs of its upper and left adjacent blocks as well as its MPMs are captured effectively, which contributes

14 most to the high detection accuracy of the proposed steganalysis algorithm.

15 Thirdly, the 4915 dimensional RoAIPM features obtained above can be directly sent into Support

16 Vector Machine (SVM) to classify stego-videos and cover-videos, outputting a detection accuracy.

17 Besides, to reduce the training time and storage space, the dimension of features can be reduced by using

18 Principal Component Analysis (PCA) method, which aims to linearly transform multiple indexes into a

19 few new comprehensive indexes. PCA is a well-known method for feature extraction by retaining low-

20 order principal components and ignoring high-order principal components, and the features of data set

21 with the larger contribution to the square difference will be maintained. The detailed principle of PCA 
realization can be searched in James V. Stone [21]. In this paper, according to the applied conditions of

2 PCA, and considering that the sample number 720 of test set is smaller than both the sample number

31520 of training set and original dimension of 4915 , we attempt to get the 720 dimensional RoAIPM

4 features by adopting PCA. Finally, SVM is also implemented for training and classification.

\section{5. Experiments}

\section{$6 \quad 5.1$ Experimental Setup}

7 In this section, experimental results will be presented to demonstrate the effectiveness and robustness of

8 the proposed steganalysis algorithm. Concrete experimental setup will be first introduced as follows:

9 (1) Video Database: Because HEVC is the state-of-art video codec standard, which is specially

10 designed for high definition videos to achieve higher coding efficiency, 22 YUV sequences (aspen, blue

11 sky, controlled burn, crowd run, ducks take off, factory, in to tree, life, old town cross, park joy,

12 pedestrian area, red kayak, riverbed, rush field cuts, rush hour, snow mint, speed bag, station, sunflower,

13 touchdown pass, tractor, west wind easy) with $1920 \times 1080$ resolution are compressed by an open source

14 software X265 encoder to establish video database. However, not all of these sequences have the same

15 frame numbers, which increases difficulty in controlling experimental variables. In order to determine

16 the length of GOP clearly, all these sequences are further divided into small sequences with 100 frames

17 each, and 112 subsequences are obtained. The details of experiment environment are listed in Table 1. 
Table 1: Environment of experiments.

\begin{tabular}{cl}
\hline & \multicolumn{1}{c}{ Values } \\
\hline Encoder & X265 (ver 2.8) \\
\hline Decoder & HM 16.7 \\
\hline Different Videos Number & 22 \\
\hline Resolution of Sequences & $1920 \times 1080$ \\
\hline Total Frames to be Encoded & 11200 \\
\hline GOP Size & 10 \\
\hline GOP Structure & IPPPIPPP .. \\
\hline QP Range & $\{15,20,25\}$ \\
\hline
\end{tabular}

(2) Steganographic Methods: To evaluate the universal detection performance of the proposed

3 steganalysis algorithm, three latest IPM-based HEVC steganography: Dong et al. [14] (denoted as Tar1

4 [14]), Dong et al. [15] (denoted as Tar2 [15]) and Wang et al. [17] (denoted as Tar3 [17]), are leveraged

5 to produce stego-videos. The payload is 0.5 , which is the common payload in the three steganography

6 methods. In our experiments, different QPs can change the partition of PUs and the embedding capacity,

7 and thus the detection performance under different embedding strengths also can be analyzed.

8 (3) Steganalytic Methods: To compare the proposed steganalysis with the current effective and

9 widely-used steganalytic methods, two latest steganalysis algorithms are also implemented to build

10 detectors: Zhao et al. [18], which designed IPMC features to detect IPM-based steganography specially,

11 and Zhai et al. [22], which proposed a universal steganalysis method in multiple domains based on the

12 consistency of motion vectors. It is worth noting that Zhao et al. [18] is currently the only one 
steganalysis against IPM-based steganography, so it is used for comparison. And Zhai et al. [22] is the

2 latest universal steganalysis of video steganography in both partition mode domain and Motion Vector

3 (MV) domain. Although the embedding modification of IPMs belongs to intra-frame steganography, the

4 change of IPMs will influence the selection of MVs and coding partition modes. Thus, we also introduce

5 Zhai et al. [22] to test its universality and effectiveness when detecting the steganography in IPM domain.

(4) Training and Classification: To improve the statistical stability of the steganalysis features, in

each experiment, all I-pictures from cover-videos or stego-videos will be randomly divided into training

8 set and testing set with the ratio of 2:1, and to ensure the preciseness of experiments, the frames from the

9 same video will not be separated. The final accuracy rate is the average of results of 20 random partition.

10 The SVM [23] is employed as classifier, and we choose polynomial kernel as the kernel function.

\section{$11 \quad 5.2$ Performance on Accuracy Rate}

12 In this section, the proposed steganalysis algorithm will be compared with another two steganalysis

13 algorithms [18, 22] described above, which are represented as Zhao et al. (2015) and Zhai et al. (2019)

14 respectively, on a common dataset to illustrate the advantage of ours. In all experiments, the proposed

15 features have been tested both before and after PCA dimension reduction. We adopt accuracy rate to

16 evaluate detection performance. Accuracy rate is defined as the ratio of predicted value, which is same

17 as actual value, to all predicted results. Table 2, Table 3 and Table 4 respectively shows the detection

18 performance of the proposed steganalysis and another two steganalysis algorithms for steganography

19 method Tar1 [14], Tar2 [15] and Tar3 [17]. The optimal experimental results are displayed in bold. 


\begin{tabular}{|c|c|c|c|c|c|}
\hline Steganography & Stegan & ysis & & QP & \\
\hline method & metl & & 15 & 20 & 25 \\
\hline \multirow[t]{4}{*}{ Tar1 [14] } & Proposed & 4915 features & $99.72 \%$ & $99.72 \%$ & $99.31 \%$ \\
\hline & steganalysis & 720 features & $92.72 \%$ & $95.83 \%$ & $96.39 \%$ \\
\hline & Zhao et al. (2015) & & $91.25 \%$ & $81.25 \%$ & $71.94 \%$ \\
\hline & Zhai et al. (2019) & & $48.06 \%$ & $50.97 \%$ & $50.97 \%$ \\
\hline
\end{tabular}

Firstly, QP is the short for quantization parameter, which can control video bit rates and reflect the

3 compression of spatial details. In view of the importance of QP for video coding, all experiments are

4 conducted under three general and representative QPs. As shown in Table 2, when Tar1 [14] is detected,

5 whether the QP is 15,20 or 25 , the highest accuracy rate is always from the proposed steganalysis.

6 It's remarkable that the accuracy rates of the proposed steganalysis against Tar1 are all above 99\% while

7 the features without PCA dimension reduction. Thus, the detection effect is stable

8 in various coding conditions. Furthermore, according to research, PUs with size of $4 \times 4$ are selected as

9 cover in Tar1 [14], and results demonstrate a strong effect of the proposed features captured only from

$104 \times 4$ PUs. In addition, because the lower the dimension of features is, the less information is retained,

11 and the training accuracy will be reduced. Together with the linearity of the model may be not high, and

12 PCA can only deal with typical linear models. Therefore, the accuracy rates turn lower while the

13 dimension of the proposed features is reduced by PCA. Even so, the detection results of the proposed

14720 dimensional features are still around $95 \%$, which are higher than the accuracies of others. On the

15 other hand, the best accuracy rate given by Zhao et al. (2015) is $91.25 \%$, and QP is 15 . The detection 
1 results of Zhai et al. (2019) are around 50\%, which means this steganalysis method is nearly invalid

2 against Tar1. Zhai et al. (2019) is mainly based on the consistency of motion vectors, and in Zhai et al.

3 (2019) the experiments were conducted for H.264/AVC videos, and thus it may be disabled while

4 detecting IPM-based HEVC steganography. Generally speaking, the universal steganalysis, Zhai et al.

5 (2019), is not suitable for detecting IPM-based steganography, which has a smaller loss of visual quality.

6 To the contrary, as discussed in Section 3, the proposed features are established by capturing the change

7 of the relationship between embedding $4 \times 4$ IPMs and the adjacent IPMs as well as the MPMs, which is

8 the fundamental distortion introduced by IPM-based HEVC steganography, and thus the proposed

9 steganalysis can always obtain the highest detection accuracy when detecting the IPM-based HEVC

10 stego-videos with various embedding strengths.

11 Table 3: Accuracy rates comparison with the three steganalysis methods for Tar2 [15].

\begin{tabular}{|c|c|c|c|c|c|}
\hline Steganography & \multicolumn{2}{|c|}{ Steganalysis } & \multicolumn{3}{|c|}{$\mathbf{Q P}$} \\
\hline method & met & & 15 & 20 & 25 \\
\hline \multirow[t]{4}{*}{$\operatorname{Tar} 2[15]$} & Proposed & 4915 features & $98.33 \%$ & $98.89 \%$ & $99.44 \%$ \\
\hline & steganalysis & 720 features & $97.78 \%$ & $96.39 \%$ & $93.33 \%$ \\
\hline & Zhao et al. (2015) & & $91.94 \%$ & $85.83 \%$ & $78.19 \%$ \\
\hline & Zhai et al. (2019) & & $48.89 \%$ & $48.75 \%$ & $47.36 \%$ \\
\hline
\end{tabular}

Then, Table 3 presents the performance results of the three steganalysis methods against

13 steganography method Tar2 [15]. The conclusion is the same as above. Whatever the value of QP, the

14 accuracy rates of the proposed steganalysis algorithm are always the highest compared with the results

15 of another two steganalysis methods. The accuracy rates of the proposed steganalysis against Tar2 [15] 
1 are all about $99 \%$ while the dimension of features is 4915 . Besides, the detection results are respectively 97.78\%, 96.39\% and 93.33\% under different QPs of 15, 20 and 25 while the features are reduced to 720

3 dimensions. Despite the decline in accuracy after PCA dimension reduction, which is due to the reasons

4 analyzed above, the proposed steganalysis still performs much better than other methods. When QP is

5 15, the accuracy rate of Zhao et al. (2015) is $91.94 \%$, and Zhao et al. (2015) shows the better results

6 against Tar2 than the results against Tar1 [14]. One major reason is that Tar2 [15] utilizes multi-sized

7 PBs to embed information instead of just $4 \times 4$ PBs used as cover in Tar1 [14], which improves the

8 embedding capacity, and thus Tar2 [15] is easier to detect. Besides, it is not hard to find that, when QP

9 is higher, the accuracy rate of Zhao et al. (2015) has a greater improvement compared with the results

10 against Tar1 [14]. For example, when QP is 25 , the detection results are respectively $71.94 \%$ and $78.19 \%$,

11 increasing about $7 \%$. The chief reason is that, according to the recursive procedure of the block partition

12 in HEVC, when QP increases, the number of small blocks decreases, and the number of large-size PBs

13 increases. Thus, the improvement of the capacity of Tar2 [15] will be more significant compared with

14 Tar1 [14]. The IPM Calibration (IPMC) features of Zhao et al. (2015) are not only extracted from $4 \times 4$

15 PBs but also from large-size PBs. Therefore, in contrast to the results against Tar1 [14], the accuracy rate

16 of Zhao et al. (2015) has a more growth as QP increases, but the results of the proposed steganalysis have

17 little change and are stabilized at around 99\% with 4915 dimensional features. 


\begin{tabular}{|c|c|c|c|c|c|}
\hline Steganography & Stegan & ysis & & QP & \\
\hline method & metl & & 15 & 20 & 25 \\
\hline \multirow[t]{4}{*}{ Tar3 [17] } & Proposed & 4915 features & $87.22 \%$ & $88.89 \%$ & $90.28 \%$ \\
\hline & steganalysis & 720 features & $92.33 \%$ & $95.14 \%$ & $95.97 \%$ \\
\hline & Zhao et al. (2015) & & $74.31 \%$ & $71.11 \%$ & $66.67 \%$ \\
\hline & Zhai et al. (2019) & & $50.56 \%$ & $51.11 \%$ & $46.67 \%$ \\
\hline
\end{tabular}

Finally, as shown in Table 4, although the accuracy rates of the proposed steganalysis are still the

3 highest among all detection results, the detection performances are distinctly descended compared with

4 the performance results in Table 2 and Table 3. Considering that Tar3 [17] selects partial IPMs of $4 \times 4$

5 PUs as cover, only the IPMs of non-uniform $4 \times 4$ PUs, which the four IPMs are different in one $8 \times 8 \mathrm{CU}$,

6 are used as cover, and if the current IPM is equal to one of its MPMs, it will also be excluded from the

7 cover set while the capacity of the current $\mathrm{CU}$ is sufficient [17]. Therefore, the proposed features, which

8 are captured by changing the IPMs of uniform $4 \times 4$ PUs to the adjacent IPM or the MPMs to generate

9 refilled matrixes, may have difficulty in reflecting the embedding distortion of Tar3 [17] by obtaining

10 the change of the relationship between the IPM and its MPMs or analyzing uniform $4 \times 4$ PUs. When QP

11 is 25 , the accuracy rate of the proposed steganalysis is $90.28 \%$ while the dimension of features is 4915 ,

12 but the accuracy is $95.97 \%$ with 720 dimensional features, increasing about $6 \%$. As far as we know, PCA

13 constructs new feature set by combining original features linearly, and in principal component space,

14 features will be sorted according to the degree of their variations in different categories. Thus, despite 15 the loss of little information, PCA discards the features with small contribution to classification, reducing 
1 the redundancy of features and improving the effectiveness of features. Not all $4 \times 4$ IPMs are used as

2 covers in Tar3 [17], so the RoAIPM extracted only from $4 \times 4$ PBs is more effective after dimension

3 reduction. Together with denoising effects, consequently, in some cases PCA dimension reduction can

4 improve the classification results. Besides, the detection results of Zhao et al. (2015) are just about $70 \%$.

5 Because this algorithm did not consider the uniqueness and innovation of HEVC video coding, and the

6 capacity of Tar3 [17] is lower. Therefore, the effect of Zhao et al. (2015) is greatly reduced.

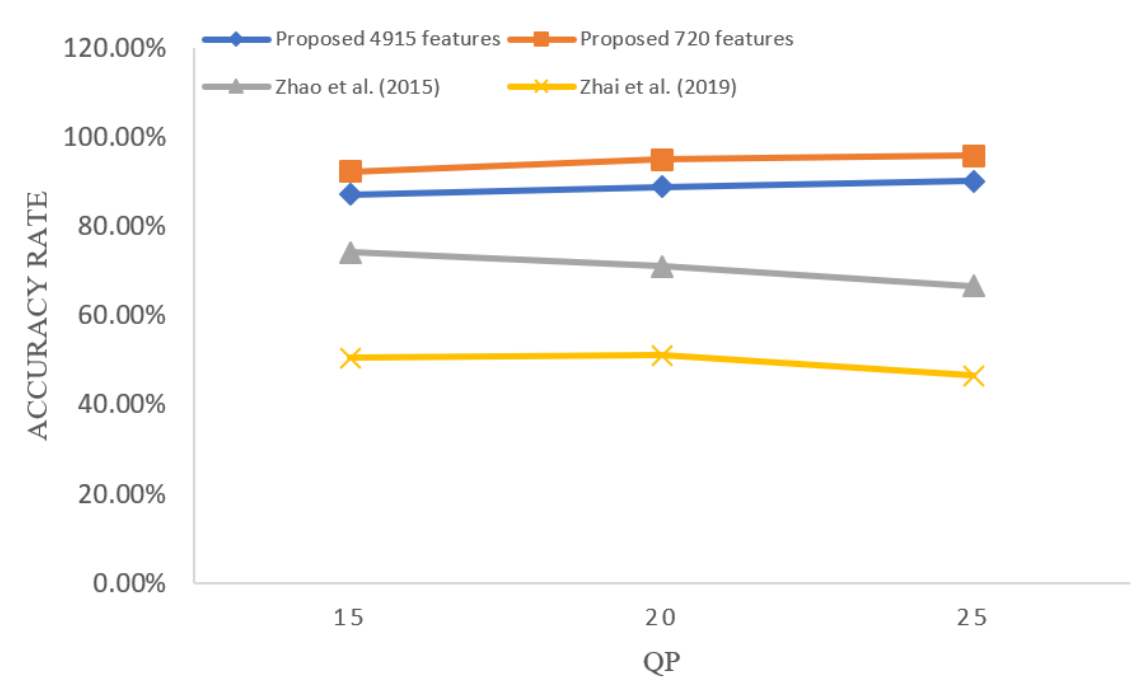

Figure 7: Trend of accuracy rates for Tar3 [17] as QP increases.

$9 \quad$ Figure 7 presents the trend of accuracy rates of different steganalysis methods as the value of QP

10 increases when detecting Tar3 [17]. It is worth noting that with the increase of QP, the accuracy rate of

11 the proposed steganalysis has an upward trend, but the accuracy rates of Zhao et al. (2015) and Zhai et

12 al. (2019) have a downward trend. According to observations, the conclusion is also applicable for Tar1

13 [14] and Tar2 [15]. For instance, in Table 4, the accuracies of the proposed steganalysis are respectively

$1487.22 \%, 88.89 \%$ and $90.28 \%$ with the features of 4915 dimensions, and the accuracies of Zhao et al.

15 (2015) are respectively 74.31\%, 71.11\% and 66.67\% when QP is 15, 20 and 25. First of all, in HEVC,

16 the number of small blocks decreases as QP increases, and the main embedding cover $4 \times 4$ PBs will 
1 decrease. Thus, Zhao et al. (2015) and Zhai et al. (2019) will perform worse as QP increases and the

2 capacity decreases. In contrast, the proposed features are extracted only from $4 \times 4 \mathrm{PBs}$, and thus the

3 change of capacity has little influence on the performance of our algorithm. In addition, the higher the

4 QP is, the coarser the quantization is, and some details are lost, which leads to the enhancement of video

5 distortion and the degradation of video quality. And the embedding distortion on the relationship between

6 the IPM and its adjacent IPMs as well as the MPMs will be more obvious. Consequently, the performance

7 results of the proposed steganalysis may be better as QP increases.

8 To summarize, the experimental results show that, regardless of whether the feature after dimension

9 reduction or the 4915 dimensional feature is provided for classifier, the proposed steganalysis algorithm

10 can both present a considerable detection accuracy when attacking several latest IPM-based HEVC

11 steganography. Under any QP, the accuracy rates are almost all above $90 \%$ and even approach $100 \%$

12 while detecting the three steganography methods. Consequently, the proposed RoAIPM features are

13 effective, performing better than existing other works.

\section{$14 \quad 5.3$ Performance on Computational Complexity}

15 In addition to the accuracy rate, the computational complexity is also a main index to consider. In order

16 to compare the computational complexity of the proposed steganalysis algorithm and other works, the

17 time spent on the feature extraction from $110 \mathrm{HEVC}$ videos is calculated, and the average time for one

18 single video is used as the final feature extraction time. The experimental facility is a computer with Inter

19 (R) Xeon (R) CPU E5-2630 v3 and 32GB memory. Experimental results are listed in Table 5, where $s$ is

20 the short for second (a unit for measuring time). The optimal experimental results are displayed in bold. 
Table 5: Feature extraction time for Tar1 [14] under different QPs.

\begin{tabular}{|c|c|c|c|}
\hline QP & Proposed steganalysis & Zhao et al. (2015) & Zhai et al. (2019) \\
\hline 15 & $78.97 \mathrm{~s}$ & $199.60 \mathrm{~s}$ & $173.27 \mathrm{~s}$ \\
\hline 20 & $70.42 \mathrm{~s}$ & $166.82 \mathrm{~s}$ & $163.46 \mathrm{~s}$ \\
\hline 25 & $63.21 \mathrm{~s}$ & $145.54 \mathrm{~s}$ & $153.84 \mathrm{~s}$ \\
\hline Average & $70.87 \mathrm{~s}$ & $170.65 \mathrm{~s}$ & $163.52 \mathrm{~s}$ \\
\hline
\end{tabular}

As presented in Table 5, when QP is higher, the feature extraction time is less. This is because

3 HEVC videos can codec faster as QP increases. Besides, the feature extraction time of the proposed

4 steganalysis is always the least compared with the results of another two steganalysis methods. For a

5 HEVC video, the extraction time is just 63.21s when QP is 25. In Zhao et al. (2015), each video needs

6 to be decompressed and compressed again, and in Zhai et al. (2019), the feature extraction needs to use

7 the encoding information of each P-picture, and thus they are more time-consuming. In conclusion, the

8 proposed steganalysis achieves the lowest computational complexity compared with existing other works.

\section{6. Conclusion}

10 In this paper, a novel IPM steganalysis algorithm for HEVC videos has been proposed. Since the previous

11 literatures mainly focus on detecting H.264/AVC steganography, this paper first models the general

12 process of IPM-based HEVC steganography methods, concluding that IPM-based HEVC steganography

13 can destroy the IPM-similarity of each embedded block and its adjacent blocks. In addition, the

14 relationships of embedded IPM and the MPMs also will be influenced. Accordingly, we adopt GLCM

15 as well as its texture properties to generate the proposed RoAIPM features. 
2 algorithms, the proposed steganalysis can always give a considerable accuracy rate. Besides,

3 after dimensionality reduction, the detection performance is still much better than other methods. And

4 the feature extraction time of the proposed steganalysis is the least compared with other steganalysis.

5 Overall, our results demonstrate a strong effect and stability of the RoAIPM features, and the proposed

6 algorithm performs better than existing other works in both accuracy rate and computational complexity.

7 Moreover, universal steganalysis has become a trend, so future research could examine the blending of

8 features extracted from multiple domains.

9

10

11

12

13

14

15

16

17

18

19

20

21 


\begin{tabular}{|c|c|c|c|}
\hline HEVC & High Efficiency Video Coding & PB & Prediction Block \\
\hline IPM & Intra Prediction Mode & CTU & Coding Tree Unit \\
\hline RoAIPM & Relationship of Adjacent IPMs & $\mathbf{C U}$ & Coding Unit \\
\hline GLCM & Gray-Level Co-occurrence Matrix & IPMC & IPM Calibration \\
\hline MPM & Most Probable Mode & SVM & Support Vector Machine \\
\hline MV & Motion Vector & TU & Transform Unit \\
\hline QP & Quantization Parameter & RMD & Rough Mode Decision \\
\hline SCM & Steganographic Channel Model & HOMоAIPM & I Homogeneity of Adjacent IPMs \\
\hline PU & Prediction Unit & RDO & Rate Distortion Optimization \\
\hline STC & Syndrome-Trellis Code & ASM & Angular Second Moment \\
\hline PCA & Principal Component Analysis & GoAIPM & GLCM of adjacent IPMs \\
\hline ASMoAIPM & ASM of Adjacent IPMs & CORoAIPM & Correlation of Adjacent IPMs \\
\hline SATD Sum & of Absolute Transformed Difference & & \\
\hline
\end{tabular}

\section{Declarations}

3 Availability of Data and Materials

4 The datasets used and analyzed during the current study are available from the corresponding author on

5 reasonable request.

\section{Competing Interests}

7 The authors declare that they have no competing interests.

\section{$8 \quad$ Funding}


1 This work was supported in part by National Natural Science Foundation of China (No. 61771270, No.

2 61572320, No. 61702034), the National Key Research and Development Projects of China under Grant

3 2018YFC0830700 and Zhejiang Provincial Natural Science Foundation of China (LR20F020001).

\section{Authors' contributions}

5 HS analyzed the background of steganalysis, and modeled the process of IPM-based HEVC

6 steganography. Besides, HS proposed the novel feature and algorithm, and completed all experiments,

7 and was the major contributor in writing the manuscript. TS and ZL corrected the manuscript. All authors

8 read and approved the final manuscript.

\section{$9 \quad$ Acknowledgments}

10 Not applicable.

\section{References}

12 [1] G. J. Sullivan, J. R. Ohm, W. J. Han, and T. Wiegand, "Overview of the High Efficiency Video

13 Coding (HEVC) standard," IEEE Transactions on Circuits and Systems for Video Technology, vol. 22,

14 no. 12, pp. 1649-1668, 2013.

15 [2] H. Zhang, Y. Cao, and X. Zhao, "Motion vector-based video steganography with preserved local

16 optimality," Multimedia Tools and Applications, vol. 75, no. 21, pp. 13503-13519, 2016.

17 [3] R. Duan and D. Chen, "Video steganography algorithm uses motion vector difference as carrier,"

18 Journal of Image and Graphics, vol. 23, no. 2, pp. 163-173, 2018.

19 [4] J. Yang and S. Li, “An efficient information hiding method based on motion vector space encoding

20 for HEVC," Multimedia Tools and Applications, vol. 77, no. 10, pp. 11979-12001, 2018. 
1 [5] L. Zhai, L. Wang, and Y. Ren, "Multi-domain embedding strategies for video steganography by

2 combining partition modes and motion vectors," in IEEE International Conference on Multimedia and

3 Expo (ICME), pp. 1402-1407, Shanghai, China, July 2019.

4 [6] S. Rana, R. Kamra, and A. Sur, "Motion vector based video steganography using homogeneous block

5 selection," Multimedia Tools and Applications, vol. 79, no. 9, pp. 5881-5896, 2020.

6 [7] R. J. Mstafa and K. M. Elleithy, "A novel video steganography algorithm in DCT domain based on 7 hamming and BCH codes," in 37th IEEE Sarnoff Symposium (Sarnoff), pp. 208-213, New Jersey Inst 8 Technol, Newark, NJ, September 2016.

9 [8] R. J. Mstafa and K. M. Elleithy, “An ECC/DCT-based robust video steganography algorithm for 10 secure data communication," Journal of Cyber Security and Mobility, vol. 5, no. 3, pp. 167-194, 2017.

11 [9] Y. Cao, Y. Wang, X. Zhao, M. Zhu, and Z. Xu, "Cover block decoupling for content-adaptive H. 264 12 steganography," in 6th ACM Workshop Information Hiding Multimedia Security (IH and MMSec), pp. 13 23-30, Innsbruck, Austria, June 2018.

14 [10] M. Suresh and I. S. Sam, "High secure video steganography based on shuffling of data on least 15 significant DCT coefficients," in 2nd International Conference on Intelligent Computing and Control 16 Systems (ICICCS), pp. 877-882, Vaigai Coll Engn, Madurai, India, June 2018.

17 [11] Z. Zhu, N. Zheng, T. Qiao, and M. Xu, "Robust steganography by modifying sign of DCT 18 coefficients," IEEE Access, vol. 7, pp. 168613-168628, 2019.

19 [12] H. Zhang, Y. Cao, X. Zhao, W. Zhang, and N. Yu, "Video steganography with perturbed macroblock 20 partition," in 2nd ACM Workshop on Information Hiding and Multimedia Security, pp. 115-122, June 212014. 
1 [13] T. Shanableh, "Data hiding in MPEG video files using multivariate regression and flexible

2 macroblock ordering," IEEE Transactions on Information Forensics and Security, vol. 7, no. 2, pp. 455-

$3464,2012$.

4 [14] Y. Dong, X. Jiang, T. Sun, and D. Xu, "Coding efficiency preserving steganography based on HEVC

5 steganographic channel model," in 16th International Workshop on Digital Forensics and Watermarking

6 (IWDW), vol. 10431, pp. 149-162, Magdeburg, Germany, August 2017.

7 [15] Y. Dong, T. Sun, and X. Jiang, "A high capacity HEVC steganographic algorithm using intra

8 prediction modes in multi-sized prediction blocks," in 17th International Workshop on Digital Forensics

9 and Watermarking (IWDW), vol. 11378, pp. 233-247, Jeju, South Korea, October 2018.

10 [16] Y. Wang, Y. Cao, X. Zhao, Z. Xu, and M. Zhu, "Maintaining rate-distortion optimization for IPM-

11 based video steganography by constructing isolated channels in HEVC," in 6th ACM Workshop on

12 Information Hiding and Multimedia Security (IH and MMSec), pp. 97-107, Innsbruck, Austria, June

132018.

14 [17] J. Wang, X. Jia, X. Kang, and Y. Shi, “A cover seletion HEVC video steganography based on intra

15 prediction mode," IEEE Access, vol. 7, pp. 119393-119402, 2019.

16 [18] Y. Zhao, H. Zhang, Y. Cao, P. Wang, and X. Zhao, "Video steganalysis based on intra prediction

17 mode calibration," in 14th International Workshop on Digital Forensics and Watermarking (IWDW),

18 vol. 9569, pp. 119-133, Tokyo, Japan, October 2015.

19 [19] J. Lainema, F. Bossen, W. J. Han, J. Min, and K. Ugur, “Intra coding of the HEVC standard," IEEE

20 Transactions on Circuits and Systems for Video Technology, vol. 22, no. 12, pp. 1792-1801, 2013. 
1 [20] T. Filler, J. Judas, and J. Fridrich, "Minimizing additive distortion in steganography using syndrome-

2 trellis codes," IEEE Transactions on Information Forensics and Security, vol. 6, no. 3, pp. 920-935,

32011.

4 [21] "Principal component analysis and factor analysis," in Independent Component Analysis: A Tutorial

$5 \quad$ Introduction, ed. by J. V. Stone (MIT Press, 2004), pp.129-135.

6 [22] L. Zhai, L. Wang, and Y. Ren, "Universal detection of video steganography in multiple domains

7 based on the consistency of motion vectors," IEEE Transactions on Information Forensics and Security,

8 vol. 15, pp. 1762-1777, 2019.

9 [23] C. Chang and C. Lin, "LIBSVM: a library for support vector machines," ACM Transactions on

10 Intelligent Systems and Technology (TIST), vol. 2, no. 3, 2011. 
Figures

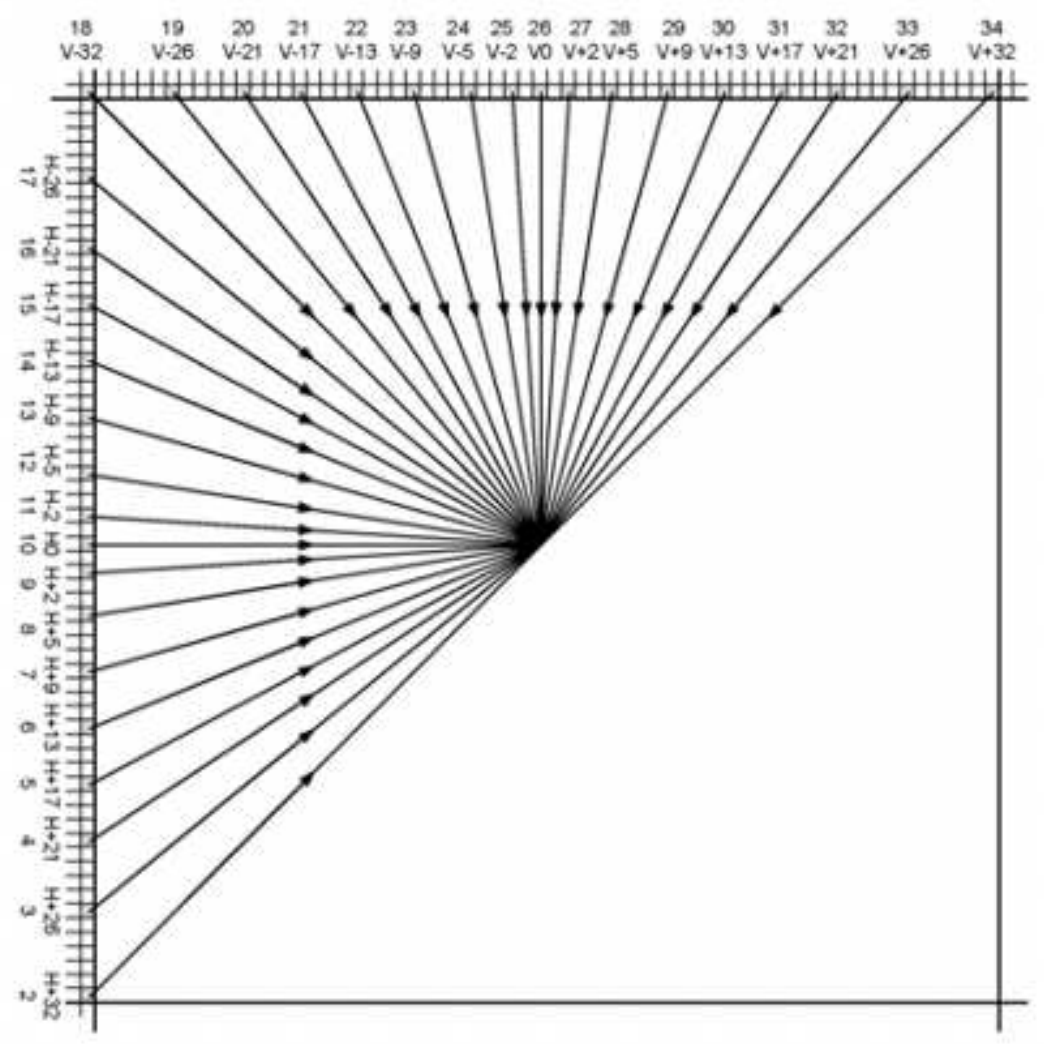

Figure 1

Angular intra-prediction modes numbered from 2 to 34 in HEVC.

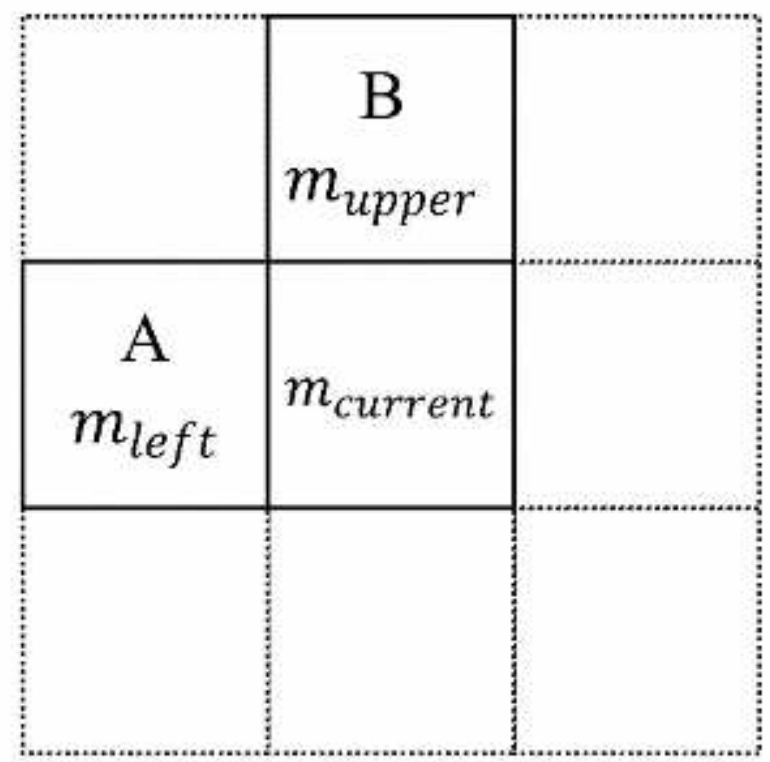

Figure 2 
Reference blocks of MPMs.

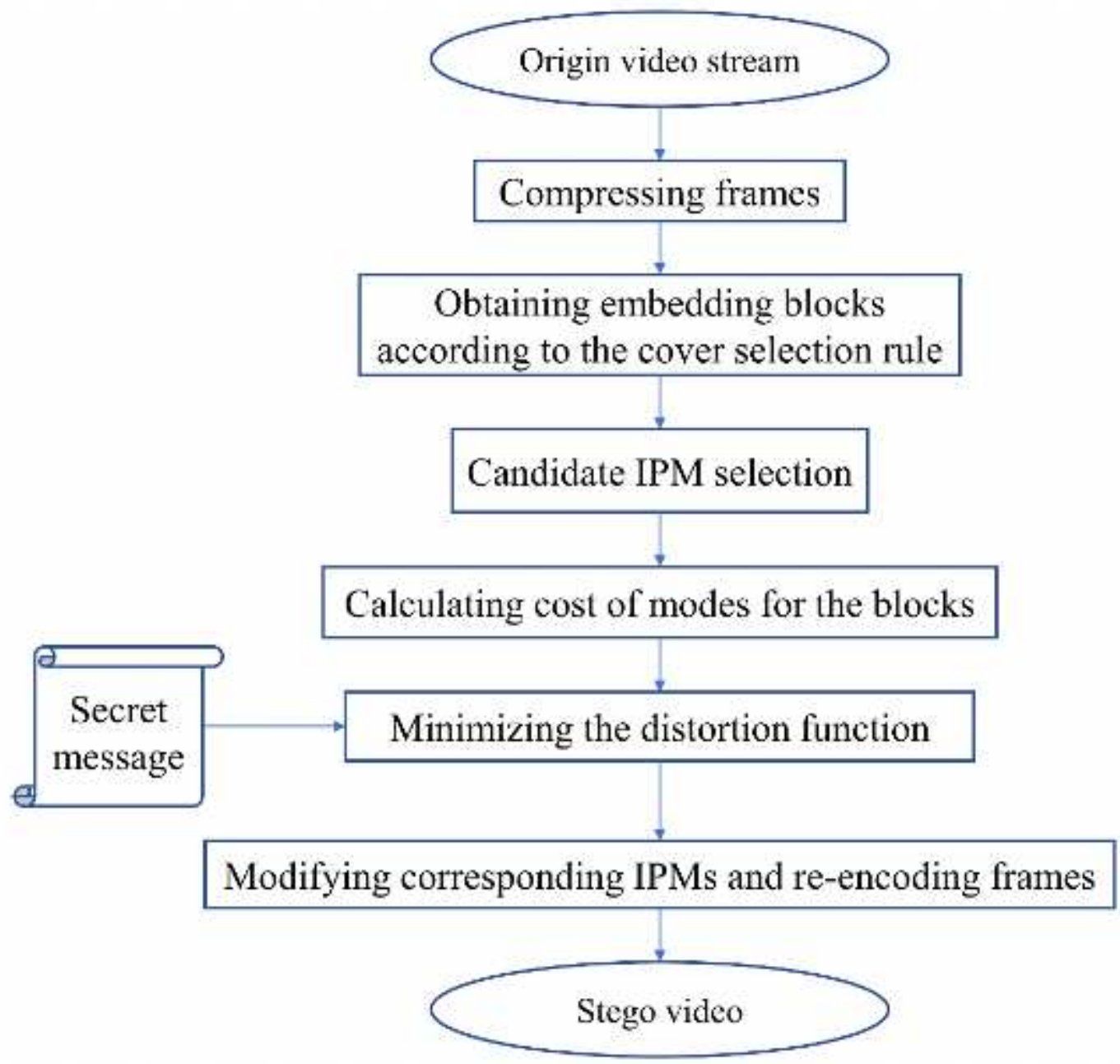

Figure 3

General embedding process of IPM-based HEVC steganography.

\begin{tabular}{|c|c|c|c|c|c|c|c|c|c|c|c|c|c|}
\hline \multirow{3}{*}{$C \frac{1}{2}$} & & & & & & & 2 & & 4 & 5 & 6 & \\
\hline & (1) & 5 & 6 & \begin{tabular}{|l|}
8 \\
\end{tabular} & \multirow{2}{*}{ GoAIPM 1} & (1) & 2 & 0 & 0 & 1 & 0 & 0 & 0 \\
\hline & 3 & 5 & 7 & 1 & & 0 & 0 & 1 & 0 & 1 & 0 & 0 & 0 \\
\hline 4 & 5 & 6 & 1 & 2 & 3 & 0 & 0 & 0 & 0 & 1 & 0 & 0 & 0 \\
\hline 8 & 5 & 1 & 2 & 5 & 4 & 0 & 0 & 0 & 0 & 1 & 0 & 0 & 0 \\
\hline & & & & & & 1 & 0 & 0 & 0 & 0 & 1 & 2 & 0 \\
\hline & & & & & 6 & 0 & 0 & 0 & 0 & 0 & 0 & 0 & 1 \\
\hline & & & & & 7 & (2) & 0 & 0 & 0 & 0 & 0 & 0 & 0 \\
\hline & & & & & 8 & 0 & 0 & 0 & 0 & 1 & 0 & 0 & 0 \\
\hline
\end{tabular}


Figure 4

GoAIPM feature generation process of matrix C.

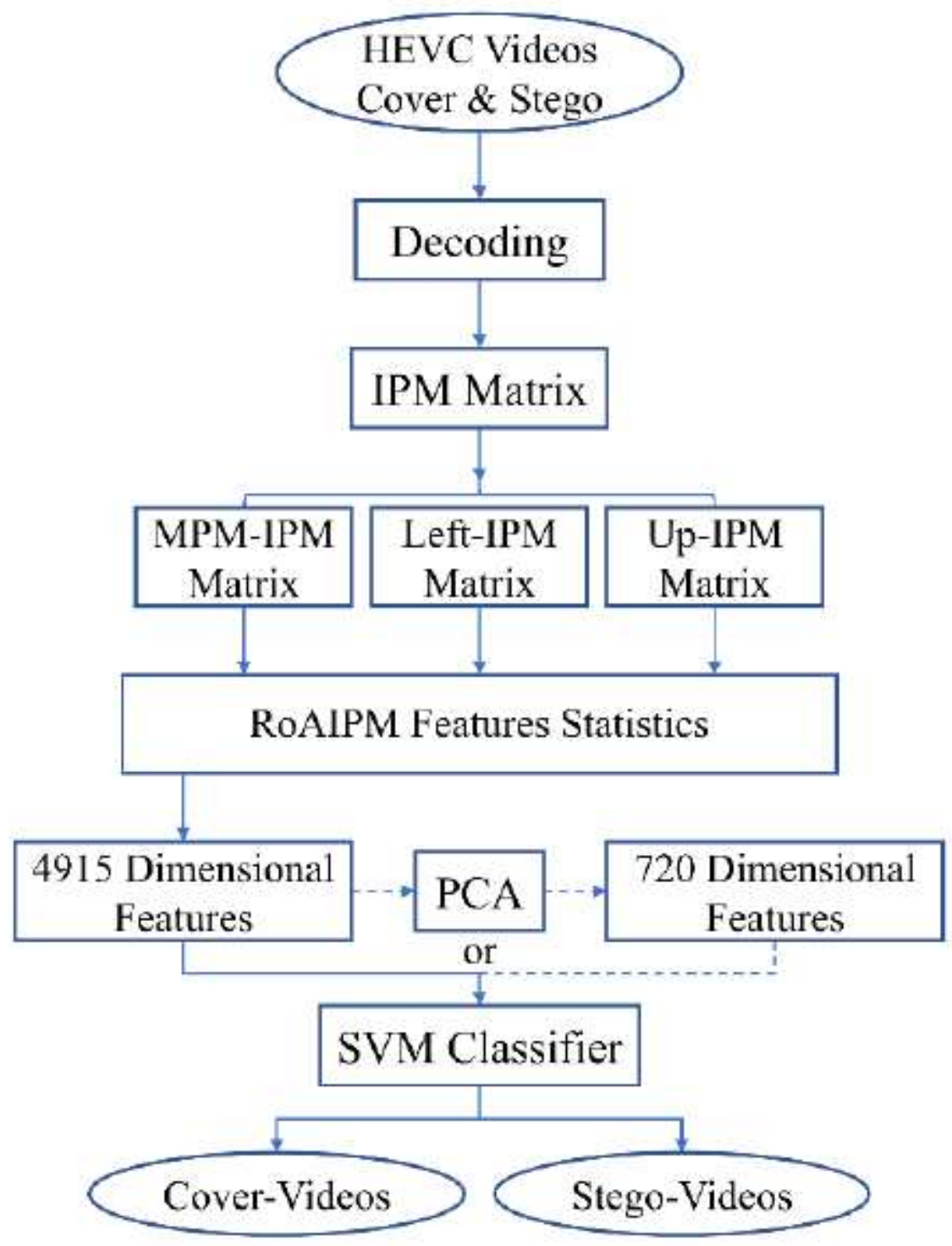

Figure 5

Framework of the proposed steganalysis algorithm. 


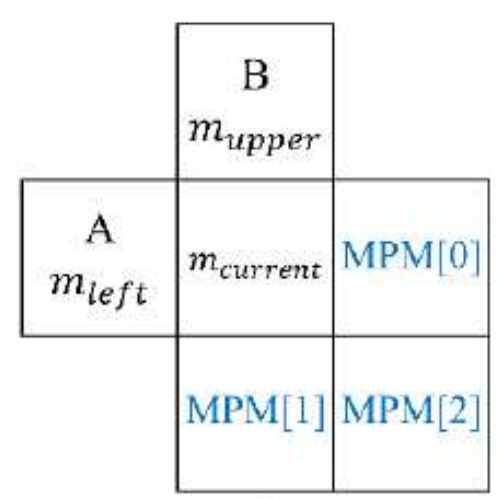

(a)

\begin{tabular}{|c|c|c|}
\hline $\begin{array}{c}\text { A } \\
m_{\text {left }}\end{array}$ & $m_{\text {current }}$ & $m_{\text {left }}$ \\
\hline & $m_{\text {current }}$ & $m_{\text {left }}$ \\
\cline { 2 - 3 } &
\end{tabular}

(b)

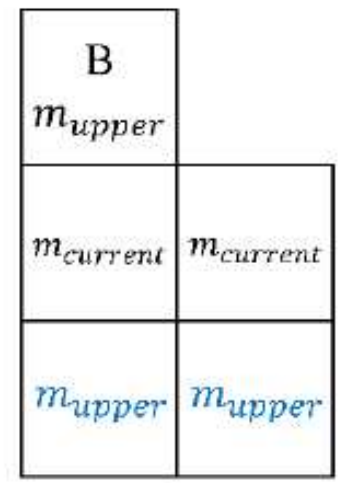

(c)

\section{Figure 6}

Filling rules of (a) MPM-IPM, (b) Left-IPM and (c) Up-IPM matrixes for uniform 4×4 PUs.

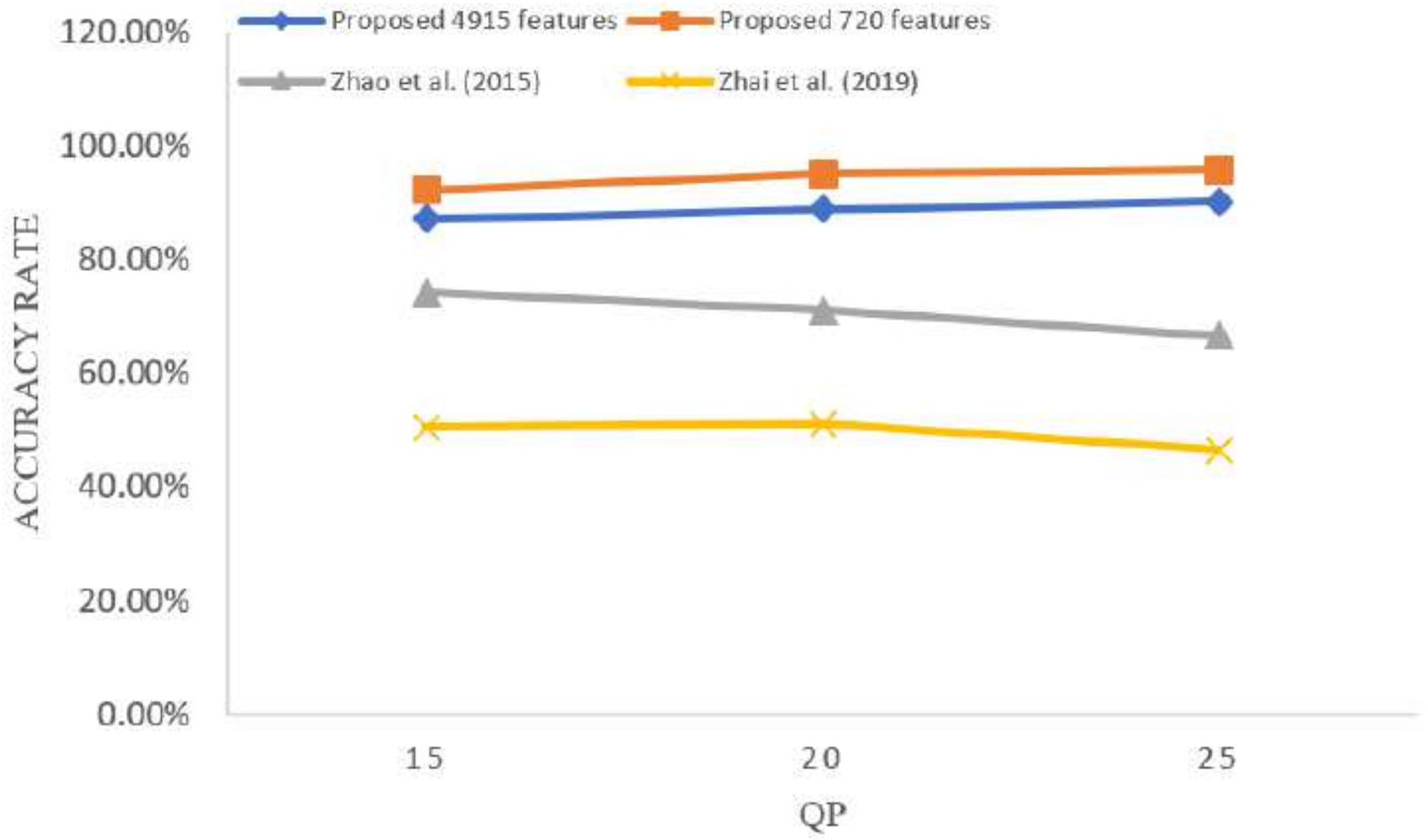

Figure 7

Trend of accuracy rates for Tar3 [17] as QP increases. 